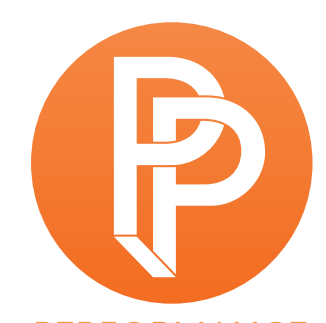

PERFORMANCE

PHILOSOPHY

\title{
IMMANENCE INPHYSICS
}

\section{TANJA TRAXLER UNIVERSITY OF VIENNA}

\section{Introduction}

Space is not only a very special research topic in physics, since it does not allow to proceed with the common method of physical research to isolate the observed object as much as possible, and thus conceptual, philosophical investigations enter the core of physics. Also, space is currently a highly relevant research topic, since it is of crucial relevance for developing a unified theory of quantum theory and general relativity. That such a theory has not been found yet is due to the fact that these two theories rely on fundamentally different conceptions of space.

Albert Einstein distinguished between two concepts of space which differ mainly in the relations between space and objects, which are (a) space as the positional quality of the world of material objects; and (b) space as a container of all material objects (cf. Jammer (1954) 1993, xv).

In concept (a) space is inconceivable without material objects and therefore objects are in a certain sense superior to space, whereas in case (b) an object can only be conceived as existing in space and thereby space appears as a reality superior to the material world.

The common structure to present the history of space in physics is a division into absolute and relative concepts of space. This is a reasonable approach, but as numerous authors have noted, this persistent classification has become a trap for the inquiry of space concepts. Therefore it seems more fruitful to re-read the history of space concepts with respect to another framework which shall be presented here, namely immanent and transcendent concepts of space. Here, 
transcendent conceptions of space refer to Einstein's concept (b), while the notion of immanence will be developed by referring to concept (a).

Both, transcendence and immanence, have a long history as philosophical concepts which have been applied to many different regimes, reaching from theological discourses to artistic researchnot so much though in the context of space studies. Concerning space, the concepts of absolute and relative have been much more dominant in the past centuries. As a consequence of their permanent reworking, at the current stage these concepts seem too outworn to expect a new account for the conceptualization of space.

\section{Complementary instead of contradictory concepts of space}

Trapped in a century-long debate, it seems that absolute and relative accounts of space are rather a contradictory than a complementary conceptual framework. Complementarity was developed by Niels Bohr, and even though the importance of the concept for the interpretation of quantum theory is widely acknowledged, it should not be left out here that it is indeed a concept that is hard to grasp; its interpretation is an ongoing subject in literature. A prominent critique came from Einstein who complained that, "despite much effort" he had expended on Bohr's principle of complementarity, he was "unable to achieve [a] sharp formulation" of it (cf. Schilpp 2000).

Bohr first introduced the concept of complementarity in a lecture at the International Physics Congress held in Como, Italy, in 1927. ${ }^{1}$ It was published briefly afterwards, in German in Naturwissenschaften and in English in Nature. In this publication Bohr starts with the quantum postulate-a principle going back to the Bohr-Sommerfeld quantization rules, according to which any change in action, with the units of angular momentum, must be an integral multiple of Planck's constant (cf. Saunders 2005, 421). From the quantum postulate Bohr derives that "any observation of atomic phenomena will involve an interaction with the agency of observation not to be neglected" (Bohr 1928, 580). Therefore, Bohr finds it impossible to ascribe an independent reality to the phenomena of observation. As a consequence, objects have complementary properties which cannot be measured precisely at the same time. Summing up the concept of complementarity in Bohr's words:

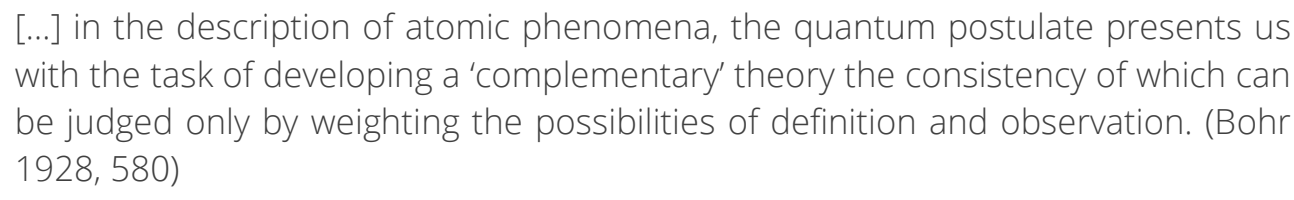

According to Bohr, it is not contradiction but complementarity which is a major principle of quantum physics, and for him there was no doubt that the concept of complementarity was important for physics and for philosophy (cf. Saunders 2005, 418). The hypothesis of this paper is that it is rather the notions of immanent and transcendent than absolute and relative that provide a complementary framework for the conceptualization of space. Here it is important to note that complementarity is not meant strictly in Bohr's sense. Whereas Bohr's principle of 
complementarity refers primarily to quantities that are being observed and measured, like position and momentum, in this paper the concept of complementarity is used in a more figurative waynot referring to strictly measurable quantities but to conceptual aspects.

The opposition of absolute and relative concepts of space seems to leave no room for complementarity: An absolute conception in which space is not changed by objects does not allow for a relative conception under any circumstances. In contrast to that, transcendent and immanent concepts of space can be understood as a complementary framework. Here, transcendent conceptions of space denote an ambient super-structure that exists prior to or independent of material phenomena, while in an immanent conception space does not exist apart from objects but is created relationally through them. In this sense, the immanent conception is closer to the relative conception, while the transcendent conception shows many similarities with the absolute conception of space without the absolute invariable character that is inconsistent with any relative or immanent account.

As a prime example of a transcendent conception of space in this paper, Isaac Newton's theories of space will be discussed as well as the mathematical framework within which Newton developed his physics: Euclidean space. On the other hand, as a role model for an immanent conception of space, Einstein's general relativity will be analyzed as well as the geometry that lies behind this theory: Riemann's differential geometry.

\section{How to take history?}

But in which ways is a historical analysis of significance for the practice of temporary research? As a philosopher of physics one is confronted with the crunch question "How do you take history?" at the very beginning: While in the disciplinary practice of physics the knowledge of the most recent scientific paradigm seems to unnecessitate the knowledge of all earlier conceptions, for philosophy the opposite seems to be true. A deep understanding of recent concepts can only be obtained by the knowledge of their etymological history.

This is to say that, unlike philosophy, physics is a non-historic discipline in the sense that in the practice of research in physics historical knowledge of the concepts in use just plays a minor role. But what does this mean for philosophy of physics, where a methodology that is fundamentally based on history meets a scientific history oblivion?

In the persistent commitment of the progressiveness of its endeavor, science permanently leaves its past behind. In its constant hunt for fresh results, science tends to forget that it is just the minority of scientific 'truths' that sustain preserved. But this non-historic and short-sufficient tendency of science shall not be accepted uncritically; there is a need for the study and interpretation of a sort that science itself cannot supply (cf. Olby, Cantor, Christie and Hodge 1990, xix). 
Even if physicists are not always aware of it in the practice of experimentation and calculations, physics being a part of science is a project that humans have pursued across generations and throughout centuries. Arkady Plotnitsky referred to the history of science as trajectories, ${ }^{2}$ some of them short, some of them longer, and makes the point that every physical theory has its trajectory:

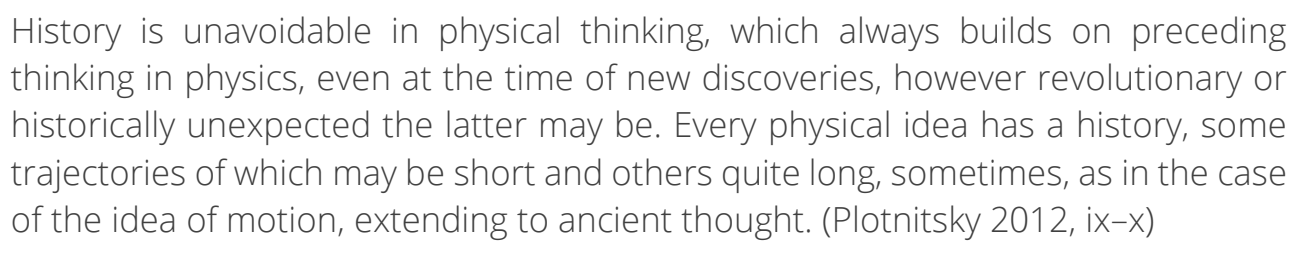

To put it differently: an argument for the history of science can be drawn from the explanatory power of the past in regard to the present (cf. Olby et al. 1990, xviii). The scientist is dependent on means that allow for overseeing a big stretch of time, as the scientific results should not just hold for the moment but in the best case quite generally. And "because the present is a short-run affair and the future is inaccessible, the past is the only long run to which we have access" (Olby et al. 1990, xviii).

Of course, the history analysis should not assume that space or place have the same meaning e.g. in Newton's physics as in Einstein's, but the relevance of Einstein's conception of space and place gains relevance through the considerations of the critique it does to Newton.

Considering the relations of Newtonian mechanics with Einstein's general relativity, it is crucial to address that Einstein did not do away with Newton's theory-as it is often wrongly claimed. On the contrary, Newton's theory is still an element of Einstein's theory for the limit of small velocities (compared to the speed of light) and average masses (not as small as an atom, not as huge as a planet). In this sense, the transition from Newton to Einstein does not appear as a strict paradigm shift in the context of which the former scientific paradigm is entirely replaced by the newer paradigm, rather the new theory highlights the boundaries of the older theory.

In his foreword to Jammer's treatise, Albert Einstein expressed his gratefulness as a scientist to historical investigations of scientific concepts. According to him, the historian is capable of correcting views of the scientist that are of "purely intuitive origin" (Jammer (1954) 1993, xiv). The full quotation of his statement shall be proposed as a prologue to the following investigation:

\footnotetext{
If two different authors use the words "red," "hard," or "disappointed," no one doubts that they mean approximately the same thing, because these words are connected with elementary experiences in a manner which is difficult to misinterpret. But in the case of words such as "place" or "space," whose relation with psychological experience is less direct, there exists a far reaching uncertainty of interpretation. The historian attempts to overcome such uncertainty by comparison of the texts, and by taking into account the picture, constructed from literature, of the cultural stock of the epoch in question. The scientist of the present, however, is not primarily trained or oriented as a historian; he is not capable of forming nor willing to form his views on the origin of the fundamental concepts in this manner. He is more inclined to allow his views on the manner in which the
} 
relevant concepts might have been formed, to arise intuitively from his rudimentary knowledge of the achievements of science in the different epochs of history. He will however, be grateful to the historian if the latter can convincingly correct such views of purely intuitive origin. (Ibid., xiv)

\section{Transcendent concepts of space}

In our modern scientific language, characterizations of space as continuous, quantized, homogeneous, isotropic, flat or curved seem self-evident. However, we have to keep in mind that these qualities are not as such directly accessible to our sensual perception. Therefore, they are the result of a long process of abstraction which had its beginnings even before ancient Greek philosophy (ibid., 138).

With the formalization of Euclid in the sixth century B.C. the conception of space was based on geometry. Euclid described his mathematical system in a textbook on geometry called Elements, in which he started by assuming a small set of axioms and derived a couple of propositions from them. Since the Elements are in large parts a collection of results that had been obtained by earlier mathematicians, some of the best known propositions of the book are not findings by Euclid himself: e. g. according to the Pythagorean theorem, the sum of the areas of the two squares on the legs of a right triangle equals the area of the square on the hypotenuse; or as Thales' theorem states: if $A, B$ and $C$ denote the vertices of a triangle and $A C$ is a diameter, then the angle at $B$ is a right-angle.

According to Euclid, space is infinite, homogeneous, which means that any point in it is like any other and isotropic, therefore it looks the same from all directions (cf. Riemann and Jost 2016, 9).

\section{Newton's transcendent conception of space}

A physical theory that is based on Euclid's geometry is Newtonian mechanics. In the understanding of Isaac Newton, physical space is fundamentally independent of the bodies ${ }^{3}$ by which it is occupied. Also, space gains in ontological priority over bodies: Neither do bodies curve or stretch areas of space which they inhabit, nor are they a precondition for the existence of space. For Newton, Euclidean space served as an invariant container in which objects were moving, stimulated by forces (cf. ibid., 12). Through the formulation of classical mechanics in his Philosophiae Naturalis Principia Mathematica (first published in 1687), Newton prefigured not only the approach of modern natural sciences but also inspired philosophers. Most remarkable about his mechanics is that it was the first scientific theory which explained the motions of the planets by the same means and in the same framework as the motions of bodies on earth.

Therefore, still today Newton's mechanics hold as a prime example for the economic character of scientific theories: Newton's laws of gravitation are capable of putting together very different phenomena, like the famous apple falling from a tree to the motion of planets, and of describing them by means of a few simple principles. 
To reduce different phenomena to a few basic principles, Newton came up with the idea that gravity can, in principle, be understood as a force. This becomes explicit in Section XI of Newton's Principia where he made the point that "attractions are made towards bodies" (Newton (1687) $1846,218)$, which is gravity. In his preface to the second edition of the Principia, Roger Cotes acknowledged Newton's concept of gravity: “This is that incomparably best way of philosophizing, which our renowned author most justly embraced before the rest; and thought alone worthy to be cultivated and adorned by his excellent labours. [...] That the virtue of gravity of found in all bodies, others suspected, or imagined before him; but he was the only and the first philosopher that could demonstrate it from appearances, and make it a solid foundation to the most noble speculations" (Newton 1729, xvi).

Newton dominated the understanding of space from the 17th century on, until it was pushed aside by Einstein's general relativity theory at the beginning of the 20th century. Nonetheless, Newton's mechanics has remained to be of major importance and is referred to in debates still today. ${ }^{4}$

In the translation of Andrew Motte of the Principia from Latin to English, Newton's laws of mechanics read as follows (Newton (1687) 1846, 19-20): LAW I. Every body perseveres in its state of rest, or of uniform motion in a right line,
unless it is compelled to change that state by forces impressed thereon.

LAW II. The alteration of motion is ever proportional to the motive force impressed; and is made in the direction of the right line in which that force is impressed.

\section{LAW III. To every action there is always opposed an equal reaction: or the mutual actions of two bodies upon each other are always equal, and directed to contrary parts.}

Interestingly, none of these three laws of Newtonian mechanics explicitly requires or involves an absolute conception of space. Rather it seems that it is more Newton's personal choice to propose the absolute character of space-it is not an argument that follows straightforward from the laws of his mechanics.

That the absolute character of Newton's space is questionable can also be seen with another argument: At the beginning of the $20^{\text {th }}$ century Einstein's theory of general relativity showed that Newton's theory is not wrong altogether but works just in a relatively small regime, namely for velocities that are much smaller than the speed of light and for masses that are much larger than atoms but much smaller than planets. Consequently, given that absolute and relative space are two mutually exclusive concepts that could not gradually transform into one another, one runs into a contradiction when considering a body like a rocket that gradually shifts from a Newtonian motion to a speed that can only be properly framed within the theory of relativity. This gradual shift would require a gradual transformation from an absolute account of space to a relative one, which is not impossible by the very definition of absolute and relative space. 
Rather than the questionable absolute character of Newtonian space, what is interesting for this paper is its obvious transcendent character: In Newton's understanding space functions as an ambient super-structure that transcends material bodies-a conception that goes back to ancient thinkers like Euclid and can also be found in contemporary scientific models of space.

The transcendent conception of space which lies behind Newton's physics is Euclidean geometry, which Newton used powerfully to mathematically depict the physical universe. Unlike Riemannian geometry, Euclidean geometry is always based on the assumption that a further dimension is needed to describe the geometry of an object: a one-dimensional object like a point calls for a twodimensional coordinate system to be described in Euclidean geometry, a two-dimensional object requires a three-dimensional coordinate system and so on and so forth.

The transcendent character of Newton's conception of space becomes apparent in his crucial assumption that space is fundamentally distinct from matter and time. From this Newton drew the conclusion of "absolute space". For an illustration of Newton's concept of a fixed and absolute space see Fig.1.

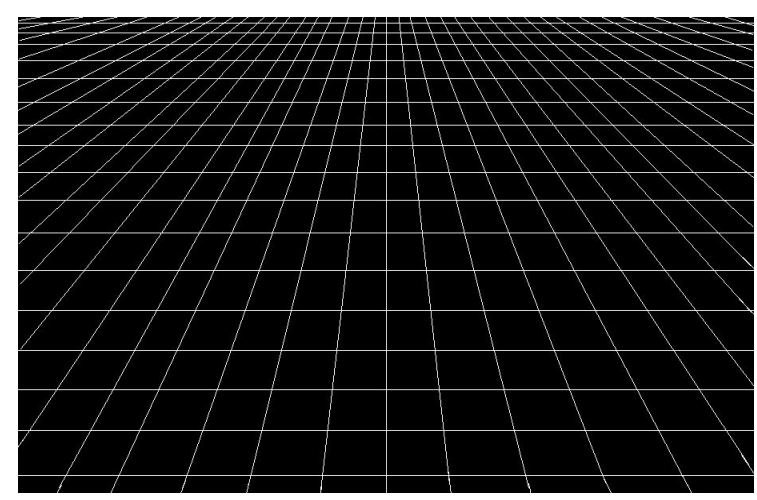

(a) Without massive bodies.

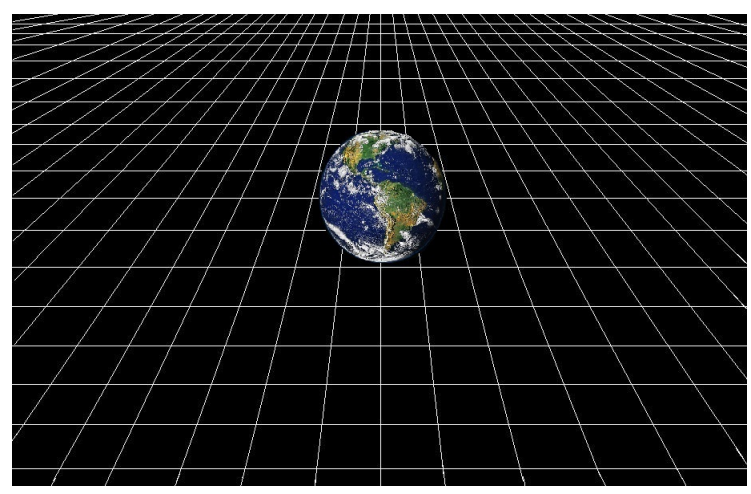

(b) With massive body.

Figure 1: In Newton's conception of absolute space the structure of space does not change depending on whether it is filled with bodies (b) or empty (a). Credit: Tanja Traxler

In the Scholium of his Principia Newton distinguishes absolute space from the ways of measuring space, which he calls "relative spaces". Absolute space "remains always similar and immovable" (ibid., 9) without relation to external bodies ${ }^{5}$-which fulfills the properties of a transcendent conception as outlined above. This type of space is not accessible by observation but a purely theoretical entity. Thus, the structure of absolute space can be denoted as an absolute reference frame, an absolute structure that necessarily underlies physical events and thus cannot be abandoned. Speaking in mathematical terms, this immovable structure was at that time assumed to be that of the three-dimensional Euclidean space, traditionally denoted with the sign $E^{3}$ (cf. Earman 1989, 9).

In contrast to that, for Newton, relative spaces are "some movable dimension or measure" (Newton $(1687) 1846,77)$ of absolute space. In modern scientific language, Newton's relative spaces come 
close to a scale. They are thus the means that makes spatiality accessible by observation. Relative spaces are defined with reference to bodies, so they are likely to be in motion (cf. Rynasiewicz 2014)-very much in contrast to absolute space.

Newton's absolute space remains unchanged by the presence of an observer (a hypothesis that will be questioned by quantum mechanics centuries later) or objects (a hypothesis that will be attacked by Einstein's general relativity theory). According to Newton, any moving body moves relatively to absolute space.

In the Scholium Newton sketches an argument for the absoluteness of space in paragraph VI. This argument is based on the assumption that space can be treated analogously to time. As Newton states, just like parts of time, parts of space will not change with respect to each other: Today will always be before tomorrow. Similarly, if parts of space were to move out of their places, they would be required to move out of themselves, which Newton denotes as being absurd: "All things are placed in time as to order of succession; and in space as to order of situation. It is from their essence or nature that they are places; and that the primary places of things should be moveable, is absurd" (Newton (1687) 1846, 79).

Before considering contemporary transcendent conceptions of space, it should be mentioned here that even though Newton's concepts of space and time have been dominant for centuries, they have faced criticism ever since. During the 17th century Christian Huygens and Gottfried Wilhelm Leibniz were Newton's most prominent opponents. While Huygens expressed his compunctions in private correspondence, Leibniz pursued public attack, to which Newton rejected to respond in public. As a result there emerged the famous Leibniz-Clarke debate, in which Samuel Clarke argued as a spokesman for Newton. Although Leibniz's ideas should prove more influential centuries later, at the time of their debate Newton won because of the superiority of his force concept (cf. Riemann and Jost 2016, 14).

Still today the discourse on space conception is dominated by a dichotomy of absolute-relational, which goes back to Newtonian thinking. According to Earman, this controversy does not just concern space but "taps some of the most fundamental concerns in the foundations of physics, metaphysics, and scientific epistemology" (Earman 1989, 2). According to him, this is a cause for both despair and hope: "[D]espair because if the absolute-relational controversy cannot be resolved without first settling the big questions of metaphysics and epistemology, it is not likely to be resolved, and hope because a way of making progress on the absolute-relational controversy can lead to progress on the big questions" (ibid., 9).

\section{Transcendent features in contemporary theories}

While the conception of space behind the general theory of relativity can be characterized as an immanent account of space, concerning quantum mechanics the issue is not so clear. For discussing this matter it is helpful to distinguish between the mathematical space of the quantum mechanical formalism, ${ }^{6}$ which is the Hilbert space, and the space concept that falls from the 
observation of quantum mechanical phenomena and will be addressed as phenomenological space in this paper.

The Hilbert space can be considered a generalization of Euclidean space: It extends the methods of algebra and calculus from the two or three dimensional Euclidean spaces to spaces with any other finite or even infinite number of dimensions. As it possesses the structure of an inner product that allows length and angle to be measured and there are enough limits in the space to allow for the use the techniques of calculus, the Hilbert space can be considered rather a transcendent than an immanent space concept, just like Euclidean space.

Also when considering the phenomenological space of quantum mechanics, the transcendent rather than the immanent character becomes prevalent-at least concerning the picture physicists draw of their experimental setups. For practical reasons, the idea is rather to start with the space that is available on an experimental table and to place there the different components that are needed for the experiment, like polarizers, filters or a laser (the transcendent conception), than to let the space of experimentation emerge from the different objects and people that are involved in the experiment (the immanent conception).

So what is transcendent space good for, after all? In this section it has been demonstrated that transcendent conceptions of space start with the postulate that space can be set as a superstructure which is not directly accessible by observation but serves as an order for material objects.

The benefits of transcendent conceptions of space are thus closely linked with the benefits John Earman identifies in absolute space: "Absolute space is a theoretical entity; this is, it is an entity not directly open to observation. It nevertheless makes good scientific sense to postulate this entity, because the explanation of various phenomena that are observable, particularly those involving rotation, calls for an absolute concept of motion, which in turn must be grounded on absolute space" (ibid., 10-11).

Analogously, it is true for transcendent conceptions of space that even though they are empirically not well-founded, they have explanatory power for certain configurations. For example, in the lab situation a picture based on a transcendent conception is much more intuitive and appropriate than a picture that was based on an immanent conception in which the space of experimentation would emerge from the involved objects.

Characteristics of this later conception will be discussed in the following section.

\section{Immanent concepts of space}

Unlike transcendent conceptions of space, in which space provides a super-structure for the organization of bodies and events that change over time, immanent conceptions of space do not rely on the presumption that space exists prior to bodies. Already in ancient Greek philosophy, 
where space appears as a subject of intellectual inquiry, both transcendent and immanent can be found.

Emancipating from the considerations of primitive thought, where concepts of space were not treated independently of the experience of space, also in Pythagorean geometrization, space had not yet any physical implication apart from serving as the limiting agent between different bodies (cf. Jammer (1954) 1993, 9). As such, space is what separates different bodies. The maxim is: Every body occupies a certain space (cf. Nikolaou 1998, 19-20).

Here the concept of space is still confounded with that of matter, which can be seen from the fact that in early Pythagorean philosophy space is still called pneuma apeiron and only occasionally kenon (cf. Jammer (1954) 1993, 9) Consisting of pneuma ( $\pi v \varepsilon \cup \mu a$ ), which is the ancient Greek word for 'breath', in a more religious context it can also be used as 'spirit' or 'soul', and apeiron (arcเpov), meaning 'unlimited' or 'infinite', according to Jammer pneuma apeiron can be understood as airan unlimited medium of breath, whereas kenon can be translated as 'void' (coming from the Greek

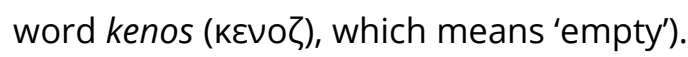

At the times of Newton an important immanent conception of space was proposed by Leibniz. As a leading figure of the Scientific Revolution, the German mathematician, philosopher and political adviser left a huge intellectual legacy, ranging from political recommendations concerning the Viennese street lighting system of those days, a recipe for takeaway food for soldiers (in reference to that the German enterprise Bahlsen markets biscuits as 'Leibniz's Butterkeks' still today) to the refinement of the binary number systems on which modern information technology is based and important philosophical oeuvres like Monadology and Theodicy.

Concerning the conception of space, in contrast to Newton, for Leibniz space is not absolute but relative-but in a way which is fundamentally different from Newton's conception of relative spaces.

What does that mean? In Newton's conception of absolute space, space is understood as a container, a box that may be occupied by bodies. Their motion is then defined in relation to this absolute space. Leibniz's conception of space runs opposite to this: In his understanding, space yields as the structure of the relations of bodies. So to say, space is the order of the coexistence of objects. This implies that the existence of space depends on the existence of bodies-a thought that is entirely alien to Newton's conception.

\section{Riemann's immanent geometry}

As a role model for an immanent conception of space, in this paper the focus is on differential geometry developed by Carl Friedrich Gauss and Georg Bernhard Riemann. Not only philosophers like Henri Bergson and Gilles Deleuze have referred to Riemannian space in their thinking, but it is also the mathematical foundation of Einstein's general relativity theory. 
In opposition to analytical geometry that bases on a coordinate system and uses techniques of algebra and analysis, differential geometry uses the techniques of differential and integral calculus to study geometric issues. While Gauss is limited to two dimensional considerations, his student Riemann posed the question of the extension of differential geometry to higher dimensions.

Departing from the surface studies of Leonhard Euler and Gaspard Monge, Gauss started his studies with the traditional Cartesian method and considered a curved two-dimensional surface embedded in a three-dimensional space. Considering curves on the surface and recurring to several theorems, ${ }^{7}$ Gauss could show that a surface "can be considered as a space in itself" (Kline 1990, 888). Morris Kline stresses that here one "can forget about the fact that the surface lies in a three-dimensional space" (ibid., 888), which means that the study of the two-dimensional surface must not necessarily be embedded into a higher, namely three-dimensional coordinate system.

By implementing the coordinate axes on the surface itself within a non-Euclidean geometry, "Gauss advanced the totally new concept that a surface is a space in itself" (ibid., 882). His method was further developed by his student Riemann who not just targeted the problem of three dimensions but that of surfaces containing an arbitrary number of dimensions.

More by chance than by intention, ${ }^{8}$ in his habilitation Riemann prepared a talk on the foundations of geometry. In the 16-pages manuscript entitled Über die Hypothesen, welche der Geometrie zu Grunde liegen (On the hypotheses which lie at the bases of geometry) and presenting only one formula he developed a novel type of geometry, later known as Riemannian geometry, which was to become the basis not only for the general theory of relativity but also of quantum field theory or string theory.

Departing from philosophical rather than purely mathematical considerations, Riemann transferred the global coordinate system of Cartesian space into the local coordinate description of a mathematical object called manifold (Mannigfaltigkeit) (cf. Riemann and Jost 2016, 24). As Plotnitsky points out, the concept of the manifold allows Riemann to define certain spaces as a patchwork of local spaces without "the overall space possessing the same type of structure as these local subspaces do" (Plotnitsky 2009, 192). Hereby, "these local spaces can be considered as infinitesimally Euclidean, while the overall space is, in general, not a Euclidean space" (ibid., 192).

Quite generally speaking, what Riemann achieved with his geometry was to give a generalization that includes both Euclidean and non-Euclidean geometry. For an overall space with no curvature, the Riemannian space reduces to a flat Euclidean space; for the more general case of an overall space with curvature, only the local spaces are infinitesimally Euclidean. This means that Riemannian space can be conceptualized as being transcendent only in infinitesimal regions, while the curvature of the overall space requires an immanent conceptualization.

As Riemann's geometry (see Fig. 2) is "independent of the ambient (three-dimensional) Euclidean space" (ibid., 202), it offers a mathematical model for Leibniz's theories concerning the relational character of spatiality (cf. ibid., 202). Here the immanent character of space becomes apparent: very clearly space is no longer seen as an external, pre-given super-structure where material 
objects are places, but rather it appears as a manifold whose structure emerges internally (cf. ibid., 202). In his theory of general relativity Einstein identified mass and energy as such an internal cause which curves space.

Once again, here it becomes clear that the strict opposition of an absolute and a relative concept of space becomes a

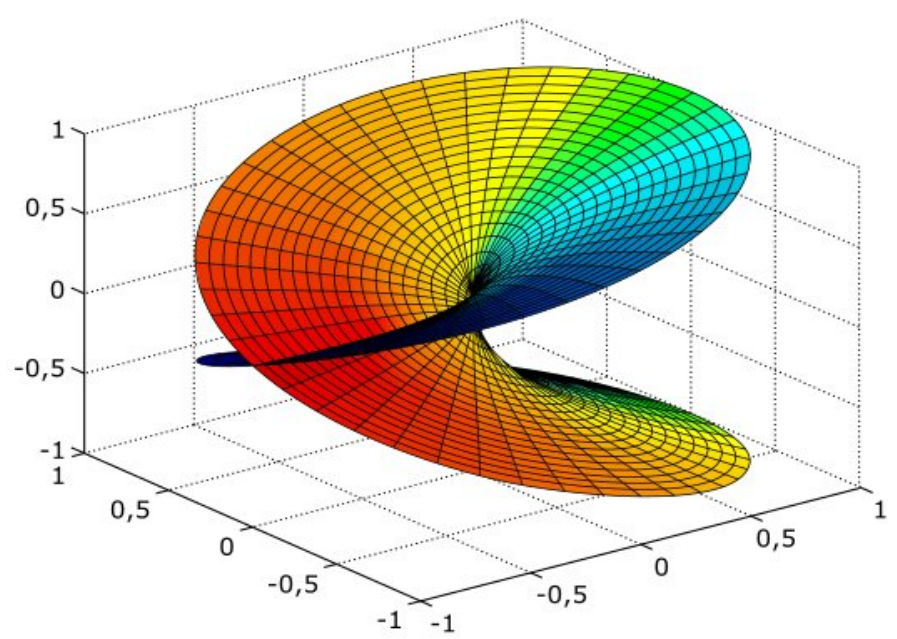
failure when it is about studying spatial configurations in detail and that it is more reasonable to Figure 2: This figure shows the Riemann surface for the function $f(z)=\sqrt{z}$.
Credit: Wikimedia Commons/Leonid 2, Open source:
https://commons.wikimedia.org/wiki/File:Riemann_sqrt.svg conceptualize space in terms of a complementary of transcendent and immanent characteristics. Considering infinitesimal pieces of a Riemann space allows for a transcendent conceptualization, while in general the immanent character is needed to describe the curvature of the overall space.

\section{Immanent concepts of space in modern physics}

Posing a realm of abstract spaces that can be studied without being embedded into higher dimensional spaces, Riemann's geometry becomes crucial for Deleuze. As Manuel DeLanda points out, eliminating a globally embedding space and viewing all spaces in purely local terms in Riemann's geometry for Deleuze "is not only a formal issue in the philosophy of mathematics but an ontological" (DeLanda 2004, 85). This becomes explicit in A Thousand Plateaus where Deleuze and Félix Guattari introduce the notion of the multiplicity which is closely connected to their concept of the rhizome as follows: "The point is that a rhizome or a multiplicity never allows itself to be overcoded, never has available a supplementary dimension over and above its number of lines" (Deleuze and Guattari 2005, 9).

60 years after Riemann had developed his geometry it turned out to serve as a foundation for Einstein's general theory of relativity. When the latter published his theory in 1915, he introduced a four-dimensional space-time that warps and curves by responding to mass and equivalently to energy (see Fig. 3). More recently, Riemann's geometry also provided the mathematical structure underlying quantum field theory and its theoretical developments in particle physics like e.g. superstring theory and quantum gravity (cf. Riemann and Jost 2016, 2) which both are major candidates for a unifying theory of quantum theory and general relativity theory. 
When comparing Fig. 1 and

Fig. 3, the differences

between Newton's

transcendent and Einstein's

immanent space become

apparent: While Newton's

space is not affected by

bodies and is conceptualized

as an ambient container in

which bodies are places, in

Einstein's picture space is

shaped relationally trough

masses and energies. Jost

concludes: "The presence of

matter thus changes the

geometry of space-time, and

acceleration is measured

now in relation to this

Riemannian geometry

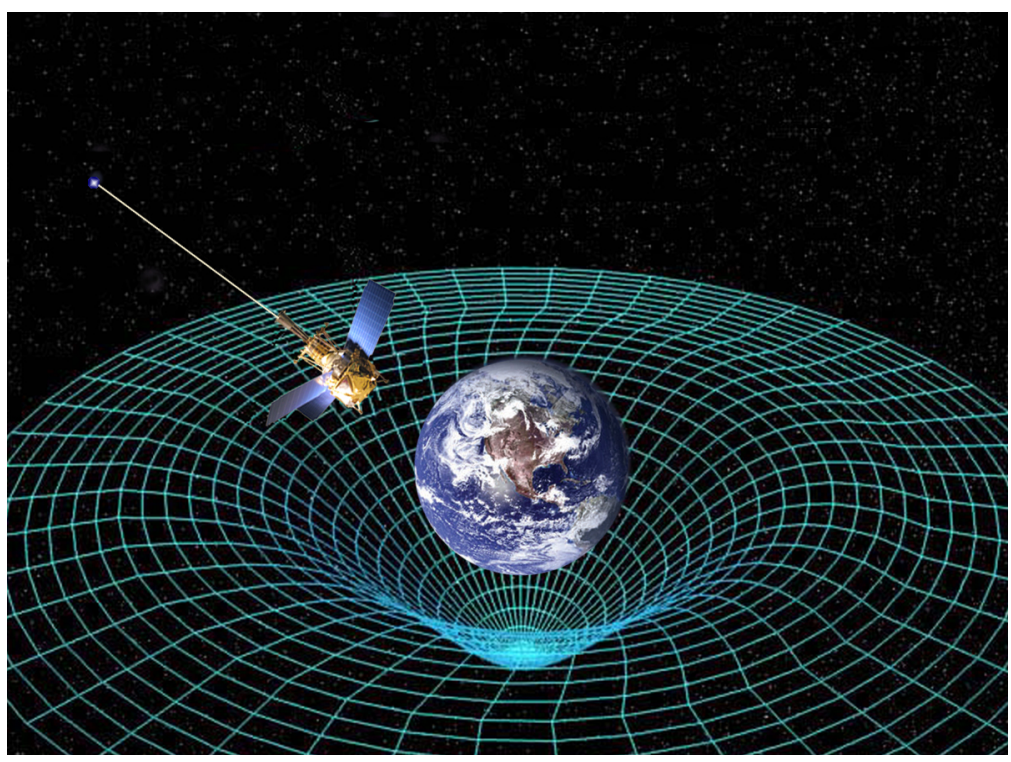

instead of an independent

absolute Euclidean one" (Riemann and Jost 2016, 130).

\section{Conclusions and Outlook: A Complementarity of transcendent and immanent} characteristics of space

Unlike absolute and relative conceptions of space, which have proven difficult to be unified within a joint conception, the framework of transcendent and immanent conceptions of space allows for a complementary conception which brings together aspects of both.

The principle of complementarity slightly adopts one of the cornerstones of ancient Greek philosophy: the principle of contradiction. In his letter-exchange with Samuel Clarke Leibniz formulated his take on the principle: "The great foundation of mathematics is the principle of contradiction or identity, i.e. that a proposition can't be true and false at the same time, so that A is A and can't be not-A. This principle is all we need to demonstrate every part of arithmetic and geometry, i.e. to demonstrate all mathematical principles" (Leibniz and Clarke (1717) 2007, 3).

In contrast to the principle of contradiction, complementarity entails that certain propositions can be true and false at the same time. More precisely, a proposition and its contradiction-in Leibniz' notation A and not-A-can be virtually true at the same time, even though both of these truths cannot be fully actualized at a certain moment under specific experimental circumstances. 
Concerning space, a complementarity framework holds virtually transcendent and immanent aspects, but depending on a certain experimental situation either transcendence or immanence is the prevalent characteristic of space.

To give an outlook, the creation of spatial concepts for a description of the complementarity of transcendent and immanent characteristics of space could prove helpful in order to properly conceptualize spatial phenomena that occur in the phenomenological space of quantum theory, like non-locality, for which a space conception that also entails the theory of general relativity is still missing. By developing such a space conception, the century-long dominance of transcendence could give way to more elaborated accounts of immanence in physics.

Notes

${ }^{1}$ Without going into detail, I want to mention here that Bohr modified the Como-version of complementarity shortly after its presentation as Arkady Plotnitsky has shown in his book Niels Bohr and complementarity.

${ }^{2}$ In physics, the notion 'trajectory' is used to describe-mathematically and geometrically - the path of a moving object through space as a function of time.

${ }^{3}$ In his Principia, Newton uses the word body equivalent to mass: "It is this quantity I mean hereafter every where under the name of Body or Mass" (Newton (1687) 1846, 2)

${ }^{4}$ As a reference see e. g. "The contemporary 20th century physics is based on a foundation which had been mainly invented by two scholars living 300 years ago in the 17th century, Newton and Leibniz, who assembled new rules for mathematics and mechanics being disparate from these known before them" (Suisky 2009, 33)

5 The insights, that space is "absolute immoveable" and incorporeal, as well as that space is a real entity even though it fits "neither of the traditional categories of substance or accident" (Rynasiewicz 2014) Newton owes to Walter Charleton's Physiologia Epicuro-Gassendo-Charltoniana: Or a Fabrick of Science Natural, upon the Hypothesis of Atoms, "Founded by Epicurus, Repaired by Petrus Gassendus, Augmented by Walter Charleton", which was published in English in 1654.

${ }^{6}$ In the mathematical formulation of quantum mechanics by John von Neumann, the pure states of a quantum mechanical system can be formulated by so-called state vectors that are living in a complex separable Hilbert space, an infinite-dimensional Euclidean space which carries a Euclidean metric structure (cf. Riemann and Jost 2016, 24).

7 For a more detailed derivation see Gauss' paper or e.g. the reformulation of Morris Kline.

${ }^{8}$ For his habilitation colloquium, Riemann had to hand in three topics and to his consternation the committee chose the topic he liked the least.

\section{Works Cited}

Bohr, Niels. 1928. "The quantum postulate and the recent development of atomic theory." Nature 121 (3050): 580590. https://doi.org/10.1038/121580a0

DeLanda, Manuel. 2004. Intensive Science and Virtual Philosophy. London: Continuum.

Deleuze, Gilles, and Félix Guattari. (1980) 2005. A Thousand Plateaus: Capitalism and schizophrenia. Minneapolis: University of Minnesota Press.

Earman, John. 1989. World Enough and Space Time: Absolute versus relational theories of space and time. Cambridge: MIT Press.

Jammer, Max. (1954) 1993. Concepts of Space: The history of theories of space in physics. 3rd enl. ed. New York: Dover. 
Kline, Morris. (1972) 1990. Mathematical Thought from Ancient to Modern Times. New York: Oxford University Press.

Leibniz, Gottfried Wilhelm and Samuel Clarke. (1717) 2007. Exchange of papers between Leibniz and Clarke. Retrieved from http://www.earlymoderntexts.com/assets/pdfs/leibniz1715_1.pdf

Newton, Isaac. 1729. The Mathematical Principles of Natural Philosophy. Volume 1. Translated by Andrew Motte. London: Benjamin Motte.

___. (1687) 1846. Newton's Principia: The mathematical principles of natural philosophy. Translated by Andrew Motte. New York: Daniel Adee.

Nikolaou, Sousanna-Maria. 1998. Die Atomlehre Demokrits und Platons Timaios: Eine vergleichende Untersuchung. Vol. Bd. 112. Stuttgart: B.G. Teubner.

Olby, Robert C., Geoffrey N. Cantor, John R. R.Christie, and M. Jonathan S. Hodge, eds. 1990. Companion to the History of Modern Science. London and New York: Routledge.

Plotnitsky, Arkady. 2009. "Bernhard Riemann." In Deleuze's Philosophical Lineage, edited by Graham Jones and Jon Roffe, 190-208. Edinburgh: Edinburgh University Press.

2012. Niels Bohr and Complementarity: An introduction. New York: Springer. https://doi.org/10.1007/978-14614-4517-3

Riemann, Bernhard, and Jürgen Jost. (1868) 2016. On the Hypotheses which Lie at the Bases of Geometry. Basel: Birkhäuser. https://doi.org/10.1007/978-3-319-26042-6

Rynasiewicz, Robert. 2014. "Newton's views on space, time, and motion." In The Stanford Encyclopedia of Philosophy (Summer 2014 Edition), edited by Edward N. Zalta. Stanford: Stanford University, Center for the Study of Language and Information, The Metaphysics Research Lab.

http://plato.stanford.edu/archives/sum2014/entries/newton-stm/

Saunders, Simon. 2005. "Complementarity and scientific rationality." Foundations of Physics 35 (3): 417-447. https://doi.org/10.1007/s10701-004-1982-X

Schilpp, Paul Arthur. 2000. Albert Einstein: Philosopher-Scientist. Vol. 7. New York: MFJ Books.

Suisky, Dieter, ed. 2009. Euler as Physicist. Berlin/Heidelberg: Springer Berlin Heidelberg.

https://doi.org/10.1007/978-3-540-74865-6

\section{Biography}

Tanja Traxler is PhD-student and university lecturer at the faculty of physics of the University of Vienna. Her interdisciplinary thesis 'Entanglement in space_-philosophical considerations on space and bodies in quantum physics' (working title), is - together with the doctoral theses of Louise Beltzung Horvath and Julia Grillmayr-part of the PhD-project 'Thinking Space' (funded by the Austrian Academy of Sciences 2012-2016 through a 'DOCteam'-grant). Being trained as a theoretical quantum physicist, in her PhD she focuses on philosophical considerations of quantum theory. She has been invited as a visiting research scholar to the University of Twente, Netherlands and to the University of California/Santa Cruz and has presented her work at more than 20 international conferences and workshops. Beside her academic career, she is working as a science editor for the Austrian newspaper Der Standard, mainly in the fields of physics and philosophy.

(C) 2017 Tanja Traxler

Except where otherwise noted, this work is licensed under a Creative Commons AttributionNonCommercial-ShareAlike 4.0 International License. 